\title{
Synthesis of 2-Aryl-4,5-diphenyl-1-(N-B-D-glucopyranosyl)- imidazoles
}

\author{
Vijay S. Taile, ${ }^{a}$ Kishor M. Hatzade, ${ }^{\mathrm{b}}$ Vikas D. Umare, ${ }^{\mathrm{a}}$ and Vishwas N. Ingle ${ }^{\mathrm{a}}$ \\ ${ }^{a}$ Department of Chemistry, Rashtrasant Tukadoji Maharaj Nagpur University, 440033 Nagpur, India \\ ${ }^{\mathrm{b}}$ Department of Chemistry, D.B. Science College, 441614 Gondia, India \\ @Corresponding authorE-mail:vijaytaile@gmail.com
}

\begin{abstract}
2-Aryl-4,5-diphenyl-1H-imidazoles (1a-g) were prepared by the reaction between aromatic $\alpha$-diketone (benzil), ammonium acetate and substituted arylaldehyde. These imidazoles were glucosylated using $\alpha$-acetobromoglucose to form 2-aryl-4,5-diphenyl-1-(N- $\beta$-D-2,3,4,6-tetra-O-acetylglucopyranosyl)imidazoles (2a-g) which were deacetylated by sodium methoxide to afford 2-aryl-4, 5-diphenyl-1-(N- $\beta$-D-glucopyranosyl)imidazoles (3a-g). All synthesized compounds were characterized spectroscopically.
\end{abstract}

Keywords: Imidazole, $\alpha$-acetobromoglucose, deacetylation, $N$-glucosides.

\section{Introduction}

A vast number of imidazoles have been reported as potential pharmacologically active compounds with antibacterial, ${ }^{[1,2]}$ antifungal, ${ }^{[3]}$ anti-inflammatory, ${ }^{[4]}$ antihistaminic ${ }^{[5]}$ and hypertensive ${ }^{[6]}$ properties. At the same time, the imidazole fragment appears in a number of naturally occurring products, among which the most important are amino acid histidine, ${ }^{[7]}$ purines, ${ }^{[8]}$ biotin, ${ }^{[9]}$ hydantoin, ${ }^{[10]}$ pilocarpine. ${ }^{[1]}$ The cellular level carbohydrates plays a key role in signaling and targeting of the organic molecule to act on the enzyme's active site. Thus, $N$-glucosides, i.e. nucleosides, has wide variety of biological activity such as antiviral, ${ }^{[12]}$ antibiotic ${ }^{[13]}$ and antineoplastic. ${ }^{[14]}$ So, in continuation of our previous works ${ }^{[15-20]}$ and keeping view of various biological activities of imidazoles and the importance of glucose moiety in the metabolism, we try to synthesize several compounds containing imidazole and glucose moiety in one framework. Herein, we reported on the synthesis of 2 -aryl-4,5-diphenyl- $1 H$-imidazoles and 2 -aryl-4,5-diphenyl-1-( $N-\beta-D$-glucopyranosyl)imidazoles.

\section{Experimental}

The melting points (m.p.) were determined using open capillary method and are uncorrected. The FT-IR spectra were recorded on Perkin-Elmer spectrophotometer using $\mathrm{KBr}$ disc. The NMR spectra recorded on Bruker DRX-300 (300 MHz FT-NMR) instrument using DMSO- $d_{6}$ as a solvent and TMS as internal standard; the chemical shifts are expressed in ppm values. EI-mass-spectra were recorded by direct insertion technique with a Hitachi Perkin Elmer RMU 6D mass spectrophotometer. Elemental analysis was carried out using the FLASH EA 1112 $\mathrm{CHN}$ analyzer, Thermo Finigin, Italy.

General procedure for the preparation of 2-aryl-4,5-diphenyl-1H-imidazoles, 1a-g. A mixture of substituted benzaldehyde ( $5 \mathrm{mmol})$, benzil $(5 \mathrm{mmol})$, ammonium acetate $(10 \mathrm{mmol})$ and glacial acetic acid $(50 \mathrm{ml})$ was refluxed for 2 hours. Then it was poured into cold water $(200 \mathrm{ml})$ and neutralized with $\mathrm{NH}_{4} \mathrm{OH}$. The solid obtained was filtered, washed with water and crystallized from alcohol.
2,4,5-Triphenyl-1H-imidazole, 1a. Yield $67 \%$, m.p. $275^{\circ} \mathrm{C},{ }^{[23]}$ $R_{\mathrm{f}}=0.48$. Found: C 85.15, H 5.45, N $9.45 \% . \mathrm{C}_{21} \mathrm{H}_{16} \mathrm{~N}_{2}$ (296.37) requires C 85.11, H 5.44, N 9.45\%. FT-IR $v_{\max } \mathrm{cm}^{-1}: 3404.4(-\mathrm{NH})$, 3028.9 (aromatic ring, str.), $1610(\mathrm{C}=\mathrm{C}), 1510\left(\mathrm{C}=\mathrm{N}\right.$, str.). ${ }^{1} \mathrm{H}$ NMR $\delta_{\mathrm{H}}$ ppm: 7.2-7.8 (m, 15H, Ar-H), $10.2\left(1 \mathrm{H},-\mathrm{NH}, \mathrm{D}_{2} \mathrm{O}\right.$ exchangeable).

2-(4-Chlorophenyl)-4,5-diphenyl-1H-imidazole, 1 b. Yield 65 \%, m.p. $250{ }^{\circ} \mathrm{C},{ }^{[24]} R_{\mathrm{f}}=0.32$. Found: C 76.25, H 4.55, N $8.45 \%$. $\mathrm{C}_{21} \mathrm{H}_{15} \mathrm{ClN}_{2}$ (330.81) requires $\mathrm{C} 76.24, \mathrm{H} 4.57, \mathrm{Cl} 10.72, \mathrm{~N} 8.47 \%$. FT-IR $v_{\max } \mathrm{cm}^{-1}: 3445.0(-\mathrm{NH}), 3059.8$ (aromatic ring, str.), 1615 $(\mathrm{C}=\mathrm{C}), 1530\left(\mathrm{C}=\mathrm{N}\right.$, str.). ${ }^{1} \mathrm{H}$ NMR $\delta_{\mathrm{H}}$ ppm: 6.3-7.5 (m, 14H, Ar-H), $10.6\left(1 \mathrm{H},-\mathrm{NH}, \mathrm{D}_{2} \mathrm{O}\right.$ exchangeable $)$.

2-(4-Methoxyphenyl)-4,5-diphenyl-1H-imidazole, 1c. Yield $71 \%$, m.p. $230{ }^{\circ} \mathrm{C},{ }^{[23]} R_{\mathrm{f}}=0.77$. Found: C 80.97, H 5.56, N $8.60 \%$. $\mathrm{C}_{22} \mathrm{H}_{18} \mathrm{~N}_{2} \mathrm{O}(326.39)$ requires $\mathrm{C} 80.96, \mathrm{H} 5.56, \mathrm{~N} 8.58 \%$. FT-IR $v_{\text {max }}$ $\mathrm{cm}^{-1}$ : $3426.6(-\mathrm{NH}), 3025.5$ (aromatic ring, str.), 1626.5(C=C), 1545 $\left(\mathrm{C}=\mathrm{N}\right.$, str.). ${ }^{1} \mathrm{H}$ NMR $\delta_{\mathrm{H}} \mathrm{ppm}: 3.8\left(\mathrm{~s}, \mathrm{OCH}_{3}\right), 6.6-7.8(\mathrm{~m}, 14 \mathrm{H}, \mathrm{Ar}-$ $\mathrm{H}), 10.9$ (1H, - $\mathrm{NH}, \mathrm{D}_{2} \mathrm{O}$ exchangeable).

2-(3-Nitrophenyl)-4,5-diphenyl-1H-imidazole, $\quad 1 d$. Yield $68 \%$, m.p. $190^{\circ} \mathrm{C}, R_{\mathrm{f}}=0.45$. Found: C 73.90, H 5.43, N $12.30 \%$. $\mathrm{C}_{21} \mathrm{H}_{15} \mathrm{~N}_{3} \mathrm{O}_{2}$ (341.36) requires C 73.89, $\mathrm{H} 4.43, \mathrm{~N} 12.31 \%$. FT-IR $v_{\max } \mathrm{cm}^{-1}: 3454.0(-\mathrm{NH}), 3067.4$ (aromatic ring, str.), $1630.3(\mathrm{C}=\mathrm{C})$, $1525\left(\mathrm{C}=\mathrm{N}\right.$, str.). ${ }^{1} \mathrm{H}$ NMR $\delta_{\mathrm{H}}$ ppm: 6.2-7.7 (m, 14H, Ar-H), 11.4 (1H, -NH, $\mathrm{D}_{2} \mathrm{O}$ exchangeable).

2-(3,4,5-Trimethoxyphenyl)-4,5-diphenyl-1H-imidazole, $\mathbf{1 e}$. Yield $54 \%$, m.p. $220^{\circ} \mathrm{C}, R_{\mathrm{f}}=0.65$. Found: C 74.59, H 5.73, N 7.27 $\%$. $\mathrm{C}_{21} \mathrm{H}_{15} \mathrm{~N}_{3} \mathrm{O}_{2}$ (386.44) requires C 74.59, H 5.74, N 7.25\%. FT-IR $v_{\max } \mathrm{cm}^{-1}: 3435.0(-\mathrm{NH}), 3030.4$ (aromatic ring, str.), $1635.5(\mathrm{C}=\mathrm{C})$, $1518\left(\mathrm{C}=\mathrm{N}\right.$, str.). ${ }^{1} \mathrm{H}$ NMR $\delta_{\mathrm{H}}$ ppm: 3.3-3.9 (m, $\left.9 \mathrm{H}, \mathrm{OCH}_{3}\right) ; 6.2-8.1$ (m, 12H, Ar-H), 10.1 (1H,-NH, $\mathrm{D}_{2} \mathrm{O}$ exchangeable).

2-(4-Fluorophenyl)-4,5-diphenyl-1H-imidazole, If. Yield $55 \%$, m.p. $235^{\circ} \mathrm{C}, R_{\mathrm{f}}=0.45$. Found: C 80.25 , H 4.82 , N $8.92 \%$. $\mathrm{C}_{21} \mathrm{H}_{15} \mathrm{FN}_{2}$ (314.36) requires C 80.24, H 4.81, F 6.04, N $8.91 \%$. FT-IR $v_{\max } \mathrm{cm}^{-1}: 3420.3(-\mathrm{NH}), 3038.8$ (aromatic ring, str.), 1616.6 $(\mathrm{C}=\mathrm{C}), 1520\left(\mathrm{C}=\mathrm{N}\right.$, str.). ${ }^{1} \mathrm{H}$ NMR $\delta_{\mathrm{H}}$ ppm: 6.2-7.2 (m, 14H, Ar-H), 10.4 (1H,-NH, D $\mathrm{O}$ exchangeable).

2-(Furan-2-yl)-4,5-diphenyl-1H-imidazole, 1g. Yield 76 \%, m.p. $198^{\circ} \mathrm{C},{ }^{[24]} R_{\mathrm{f}}=0.42$. Found: C $79.71, \mathrm{H} 4.95$, N $9.79 \%$. $\mathrm{C}_{19} \mathrm{H}_{14} \mathrm{~N}_{2} \mathrm{O}$ (314.36) requires C 79.70, H 4.93, N 9.78 \%. FT-IR $v_{\max }$ $\mathrm{cm}^{-1}: 3414.5(-\mathrm{NH}), 3042.5$ (aromatic ring, str.), $1615.2(\mathrm{C}=\mathrm{C}), 1514$ $(\mathrm{C}=\mathrm{N}, \mathrm{str}.){ }^{1} \mathrm{H}$ NMR $\delta_{\mathrm{H}}$ ppm: 6.2-7.9 (m, 13H, Ar-H), $11.2(1 \mathrm{H},-$ $\mathrm{NH}, \mathrm{D}_{2} \mathrm{O}$ exchangeable).

General procedure for the preparation of 2-aryl-4,5diphenyl-1-(N- $\beta$-D-2,3,4,6-tetra-O-acetylglucopyranosyl)- 
imidazoles, 2a-g. A mixture of 2-aryl-4,5-diphenyl- $1 H$-imidazoles $(0.05 \mathrm{~mol})$ and ACBG $(5 \mathrm{~g})$ were dissolved in dioxane $(10 \mathrm{ml})$ at $100^{\circ} \mathrm{C}$ and kept at this temperature for $4 \mathrm{~h}$. The process of reaction was monitored by TLC. The solvent was removed under reduced pressure to produce 2-aryl-4,5-diphenyl-1- $(N-\beta-D-2,3,4,6$-tetra$\mathrm{O}$-acetylglucopyranosyl)imidazoles in a good yield.

2,4,5-Triphenyl-1-(N- $\beta$-D-2,3,4,6-tetra-O-acetylglucopyranosyl)imidazole, 2a. Yield $75 \%,[\alpha]_{\mathrm{D}}{ }^{30}=-8.20$ (c, 0.1, DMSO), $R_{\mathrm{f}}=0.25$. Found: $\mathrm{C} 67.10, \mathrm{H} 5.46, \mathrm{~N} 4.47 \% . \mathrm{C}_{35} \mathrm{H}_{34} \mathrm{~N}_{2} \mathrm{O}_{9}(626.65)$ requires C 67.08, H 5.47, N $4.47 \%$. FT-IR $v_{\max } \mathrm{cm}^{-1}: 2962.3(\mathrm{Ar}-$ $\mathrm{CH}), 2790$ (glucosidic $\mathrm{CH}), 1657(\mathrm{C}=\mathrm{C}), 1576.2(\mathrm{C}=\mathrm{N}),{ }^{1} \mathrm{H}$ NMR $\delta_{\mathrm{H}}$ ppm: $2.06,1.94,1.96,2.02\left(\mathrm{~s}, 3 \mathrm{H}, \mathrm{COCH}_{3}\right), 6.5(\mathrm{~d}, 1 \mathrm{H}$, anomeric proton), $6.8-8.2(\mathrm{~m}, 15 \mathrm{H}, \mathrm{Ar}-\mathrm{H})$.

2-(4-Chlorophenyl)-4,5-diphenyl-1-(N- $\beta$-D-2,3,4,6-tetra- $O$ acetylglucopyranosyl)imidazole, $2 \boldsymbol{b}$. Yield $68 \%,[\alpha]_{\mathrm{D}}^{30}=-7.10(\mathrm{c}, 0.1$, DMSO), $R_{\mathrm{f}}=0.28$. Found: C 63.60, H 5.05, N $4.26 \%$. $\mathrm{C}_{35} \mathrm{H}_{33} \mathrm{ClN}_{2} \mathrm{O}_{9}$ (661.1) requires C 63.59, H 5.03, $\mathrm{Cl} 5.36, \mathrm{~N} 4.24 \%$. FT-IR $v_{m \text { c }} \mathrm{cm}^{-1}$ : 2955.5 (Ar-CH), 2788 (glucosidic $\mathrm{CH}), 1657(\mathrm{C}=\mathrm{C}), 1568.2(\mathrm{C}=\mathrm{N})$, ${ }^{1} \mathrm{H}$ NMR $\delta_{\mathrm{H}} \mathrm{ppm}: 2.06,1.98,1.96,2.04$ (s, 3H) $\left(\mathrm{COCH}_{3}\right), 6.2$ (d, 1H, anomeric proton), $6.4-7.2$ (m, 14H, Ar-H).

2-(4-Methoxyphenyl)-4,5-diphenyl-1-(N- $\beta$-D-2,3,4,6-tetraO-acetyl-glucopyranosyl)imidazole, $2 c$. Yield $74 \%,[\alpha]{ }_{D}^{30}=$ -11.65(c, 0.1, DMSO), $R_{\mathrm{f}}=0.35$. Found: C 65.85, H 5.55, N $4.26 \%$. $\mathrm{C}_{36} \mathrm{H}_{36} \mathrm{~N}_{2} \mathrm{O}_{10}(656.58)$ requires $\mathrm{C} 65.84, \mathrm{H} 5.53, \mathrm{~N} 4.27 \%$. FT-IR $v_{\max }$ $\mathrm{cm}^{-1}: 2968.4$ (Ar-CH), 2792 (glucosidic CH), $1655(\mathrm{C}=\mathrm{C}), 1570.2$ $(\mathrm{C}=\mathrm{N}) .{ }^{1} \mathrm{H}$ NMR $\delta_{\mathrm{H}}$ ppm: 2.00, 1.98, 1.97, $2.05\left(\mathrm{~s}, 3 \mathrm{H}, \mathrm{COCH}_{3}\right), 3.8$ $\left(\mathrm{s}, \mathrm{OCH}_{3}\right), 6.4(\mathrm{~d}, 1 \mathrm{H}$, anomeric proton), 6.5-7.8 (m, 14H, Ar-H).

2-(3-Nitrophenyl)-4,5-diphenyl-1-(N- $\beta$-D-2,3,4,6-tetra-Oacetylglucopyranosyl)imidazole, $2 \boldsymbol{d}$. Yield $80 \%,[\alpha]_{\mathrm{D}}^{30}=-8.30$ (c, 0.1, DMSO), $R_{\mathrm{f}}=0.30$. Found: C 65.59, H 4.95, N $6.27 \%$. $\mathrm{C}_{35} \mathrm{H}_{33} \mathrm{~N}_{3} \mathrm{O}_{11}(671.65)$ requires $\mathrm{C} 65.59, \mathrm{H} 4.95, \mathrm{~N} 6.26 \%$. FT-IR $v_{\text {max }}$ $\mathrm{cm}^{-1}: 2980.5($ Ar-CH), 2767 (glucosidic CH), $1651(\mathrm{C}=\mathrm{C}), 1575.0$ $(\mathrm{C}=\mathrm{N}) .{ }^{1} \mathrm{H} N M R \delta_{\mathrm{H}}$ ppm: $2.00,2.02,1.95,2.00\left(\mathrm{~s}, 3 \mathrm{H}, \mathrm{COCH}_{3}\right), 6.2$ (d, 1H, anomeric proton), $6.4-7.7(\mathrm{~m}, 14 \mathrm{H}, \mathrm{Ar}-\mathrm{H})$.

2-(3,4,5-Trimethoxyphenyl)-4,5-diphenyl-1-(N- $\beta-D-2,3,4,6-$ tetra-O-acetylglucopyranosyl)imidazole, $2 \boldsymbol{e}$. Yield $72 \%,[\alpha]_{\mathrm{D}}^{30}=$ -14.15 (c, 0.1, DMSO), $R_{\mathrm{f}}=0.34$. Found: C 63.69, H 5.62, N $3.92 \%$. $\mathrm{C}_{38} \mathrm{H}_{40} \mathrm{~N}_{2} \mathrm{O}_{12}(716.73)$ requires $\mathrm{C} 63.68, \mathrm{H} 5.63$, N $3.91 \%$. FT-IR $v_{\max }$ $\mathrm{cm}^{-1}: 2975.4(\mathrm{Ar}-\mathrm{CH}), 2772$ (glucosidic $\left.\mathrm{CH}\right), 1648(\mathrm{C}=\mathrm{C}), 1569.0$ $(\mathrm{C}=\mathrm{N}) .{ }^{1} \mathrm{H}$ NMR $\delta_{\mathrm{H}}$ ppm: 2.00, 2.01, 1.97, $2.04\left(\mathrm{~s}, 3 \mathrm{H}, \mathrm{COCH}_{3}\right)$, 3.3-3.9 (m, 9H, $\left.\mathrm{OCH}_{3}\right), 6.0(\mathrm{~d}, 1 \mathrm{H}$, anomeric proton), $6.2-7.6(\mathrm{~m}$, $14 \mathrm{H}, \mathrm{Ar}-\mathrm{H})$.

2-(4-Fluorophenyl)-4,5-diphenyl-1-(N- $\beta$-D-2,3,4,6-tetraO-acetylglucopyranosyl)imidazole, $2 f$. Yield $76 \%,[\alpha]_{\mathrm{D}}^{30}=$ -6.23 (c, 0.1, DMSO), $R_{\mathrm{f}}=0.30$. Found: C 65.20, H 5.16, N 4.95 \%. $\mathrm{C}_{35} \mathrm{H}_{32} \mathrm{FN}_{2} \mathrm{O}_{9}(644.64)$ requires $\mathrm{C}$ 65.21, $\mathrm{H}$ 5.16, F 2.95, N 4.35 \%. FT-IR $v_{\max } \mathrm{cm}^{-1}: 2982.2($ Ar-CH), 2780 (glucosidic CH), 1650 $(\mathrm{C}=\mathrm{C}), 1570.0(\mathrm{C}=\mathrm{N}) .{ }^{1} \mathrm{H}$ NMR $\delta_{\mathrm{H}} \mathrm{ppm}: 1.95,2.03,1.98,2.04$ $\left(\mathrm{s}, 3 \mathrm{H}, \mathrm{COCH}_{3}\right), 5.9(\mathrm{~d}, 1 \mathrm{H}$, anomeric proton), $6.4-7.8(\mathrm{~m}, 14 \mathrm{H}$, Ar-H).

2-(Furan-2-yl)-4,5-diphenyl-1-(N- $\beta-D-2,3,4,6$-tetraO-acetylglucopyranosyl)imidazole, $2 g$. Yield $75 \%,[\alpha]_{\mathrm{D}}^{30}=$ -6.44(c, 0.1, DMSO), $R_{\mathrm{f}}=0.25$. Found: C 64.28, H 5.23, N $4.55 \%$. $\mathrm{C}_{33} \mathrm{H}_{32} \mathrm{~N}_{2} \mathrm{O}_{9}(616.61)$ requires C 64.28, H 5.23, N $4.54 \%$. FT-IR $v_{\max }$ $\mathrm{cm}^{-1}: 2976.3$ (Ar-CH), 2768 (glucosidic CH), $1646(\mathrm{C}=\mathrm{C}), 1568.0$ $(\mathrm{C}=\mathrm{N}) .{ }^{1} \mathrm{H}$ NMR $\delta_{\mathrm{H}}$ ppm: 1.98, 2.01, 1.97, $2.03\left(\mathrm{~s}, 3 \mathrm{H}, \mathrm{COCH}_{3}\right), 6.4$ (d, 1H, anomeric proton), $6.5-7.7(\mathrm{~m}, 13 \mathrm{H}, \mathrm{Ar}-\mathrm{H})$.

General procedure for the preparation of 2-aryl-4,5diphenyl-1-(N- $\beta$-D-glucopyranosyl)-imidazoles, $3 \mathbf{a - g} . \quad \mathrm{A}$ solution of 2-aryl-4,5-diphenyl-1-( $N$ - $\beta$ - $D$-2,3,4,6-tetra- $O$-acetylglucopyranosyl)imidazoles $(2 \mathrm{~g})$ in $25 \mathrm{ml}$ of dry methanol was added to $1.5 \mathrm{ml}$ of $5 \% \mathrm{CH}_{3} \mathrm{ONa}$ solution .The reaction mixture was kept at room temperature for additional $24 \mathrm{hrs}$. It was then neutralized with ion-exchange resin (Amberlite IR 120, s.d. fine, $\mathrm{H}^{+}$form) filtered and concentrated in vacuum to afford viscous, strongly hygroscopic product's form. The residue was purified by silica gel chromatography $\left(\mathrm{CHCl}_{3}, \mathrm{MeOH}, 12: 1 \mathrm{v} / \mathrm{v}\right)$ to get the titled compound.
2,4,5-Triphenyl-1-(N- $\beta$-D-glucopyranosyl)imidazole, $\quad 3 \boldsymbol{a}$. Yield $62 \%$, semi-solid, $[\alpha]_{\mathrm{D}}{ }^{30}=-20.21$ (c, 0.1, DMSO), $R_{\mathrm{f}}=0.10$. Found: $\mathrm{C} 70.73, \mathrm{H} 5.72, \mathrm{~N} 6.10 \%$. $\mathrm{C}_{27} \mathrm{H}_{26} \mathrm{~N}_{2} \mathrm{O}_{5}(458.51)$ requires C 70.73, H 5.72, N $6.11 \%$. m/z (EI-MS): 458 (10\%), 295 (100\%), 118 (3\%), 78 (2\%). FT-IR $v_{\max } \mathrm{cm}^{-1}: 3403.9$ (intramolecular -OH, broad, stretch.), 2960.2 (aromatic str.), $1611.7(\mathrm{C}=\mathrm{N}), 1248.0(\mathrm{C}-\mathrm{N})$. ${ }^{1} \mathrm{H}$ NMR $\delta_{\mathrm{H}}$ ppm: $3.9\left(1 \mathrm{H}, 2{ }^{\prime} \mathrm{H}\right), 3.2\left(\mathrm{dd}, 1 \mathrm{H}, 3^{\prime} \mathrm{H}\right), 3.6\left(1 \mathrm{H}, 4{ }^{\prime} \mathrm{H}\right)$, $3.4\left(1 \mathrm{H}, 5\right.$ 'H), $5.9\left(\mathrm{~d}, 1 \mathrm{H}, J_{1,2}=8.1 \mathrm{~Hz}, 1^{\prime} \mathrm{H}\right.$, anomeric proton), 6.2$7.5(\mathrm{H}, \mathrm{Ar}-\mathrm{H}) .{ }^{13} \mathrm{C}$ NMR $\delta$ ppm: $155,146.7,145.3,140.4,135.0$ $134.2,133.2,132.0,129.0,128.8,128.5,128.2,128.0,127.4,127.0$, $126.8,126.6,126.0,125.8,125.0,124.0,104.04,80.0,75.4,73.5$, $72.4,65.8$.

2-(4-Chlorophenyl)-4,5-diphenyl-1-(N- $\beta$-D-glucopyranosyl) imidazole, $3 \boldsymbol{b}$. Yield $65 \%$, $[\alpha]_{\mathrm{D}}{ }^{30}=-19.67$ (c, 0.1, DMSO), $R_{\mathrm{f}}=0.12$. Found: $\mathrm{C} 65.79, \mathrm{H} 5.12, \mathrm{~N} 5.69 \% . \mathrm{C}_{27} \mathrm{H}_{26} \mathrm{~N}_{2} \mathrm{O}_{5}(492.15)$ requires $\mathrm{C}$ 65.79, H 5.11, Cl 7.19, N 5.68 \%. m/z (EI-MS): 492 (8\%, base peak), 330 (28\%), 180 (31\%), 151 (12\%), 112 (14\%), 77 (15\%). FTIR $v_{\max } \mathrm{cm}^{-1}: 3414.5$ (intramolecular $-\mathrm{OH}$, broad, stretch.), 2965.0 (aromatic str.), $1620.2(\mathrm{C}=\mathrm{N}), 1250.5(\mathrm{C}-\mathrm{N}) .{ }^{1} \mathrm{H}$ NMR $\delta_{\mathrm{H}}$ ppm: 3.2 $\left(1 \mathrm{H}, 2^{\prime} \mathrm{H}\right), 3.3$ (dd, 1H, 3’H), $3.6\left(1 \mathrm{H}, 4^{\prime} \mathrm{H}\right), 3.5$ (1H, 5'H). 6.3 (d, $1 \mathrm{H}, J_{12}=8.0 \mathrm{~Hz}, 1^{\prime} \mathrm{H}$, anomeric proton), $6.5-7.8(\mathrm{H}, \mathrm{Ar}-\mathrm{H}) .{ }^{13} \mathrm{C}$ NMR $\delta_{\mathrm{c}}$ ppm: $154,150.4,148.8,145.2,140.2,138.0,136.0,135.5$, $134.1,133.0,129.5,129.0,128.8,128.5,127.8,127.0,126.9,126.2$, 125.6, 125.3, 125.0, 124.0, 102.05, 84.0, 76.2, 75.0, 65.0.

2-(4-Methoxyphenyl)-4,5-diphenyl-1-(N- $\beta$-D-glucopyranosyl)imidazole, $3 c$. Yield $68 \%,[\alpha]_{\mathrm{D}}^{30}=-29.21$ (c, 0.1, DMSO), $R_{\mathrm{f}}=0.14$. Found: $\mathrm{C} 68.85, \mathrm{H} 5.80, \mathrm{~N} 5.75 \% . \mathrm{C}_{28} \mathrm{H}_{28} \mathrm{~N}_{2} \mathrm{O}_{6}(488.53)$ requires C 68.84, H 5.78, N $5.73 \% . m / z$ (EI-MS): $489\left(30 \%, \mathrm{M}^{+}\right)$, 325 (100\%, base peak), 295 (24\%), 178 (15\%), 78 (12\%). FTIR $v_{\max } \mathrm{cm}^{-1}: 3438.2$ (intramolecular $-\mathrm{OH}$, broad, stretch.), 2954.1 (aromatic str.), $1626.0(\mathrm{C}=\mathrm{N}), 1245.2(\mathrm{C}-\mathrm{N}) .{ }^{1} \mathrm{H}$ NMR $\delta_{\mathrm{H}}$ ppm: 3.0 $\left(1 \mathrm{H}, 2^{\prime} \mathrm{H}\right), 3.4\left(\mathrm{dd}, 1 \mathrm{H}, 3^{\prime} \mathrm{H}\right), 3.2\left(1 \mathrm{H}, 4^{\prime} \mathrm{H}\right), 3.6\left(1 \mathrm{H}, 5^{\prime} \mathrm{H}\right), 4.0(\mathrm{~s}$, $\left.\mathrm{OCH}_{3}\right), 5.9\left(\mathrm{~d}, 1 \mathrm{H}, J_{1,2}=7.8 \mathrm{~Hz}, 1\right.$ 'H, anomeric proton), 6.0-7.5 (H, Ar-H). ${ }^{13} \mathrm{C}$ NMR $\delta_{c}$ ppm: 154, 152.4, 147.3, 146.2, 144.0,139.0, 137.5, 136.5, 135.3, 133.2, 130.2, 129.4, 129.0, 128.3, 127.8, 127.2, $126.0,125.4,125.0,124.8,124.4,104.2,90.2,85.0,76.0,75.8$, $65.2,59.2$

2-(3-Nitrophenyl)-4,5-diphenyl-1-(N- $\beta$-D-glucopyranosyl)imidazole, 3d. Yield $64 \%,[\alpha]_{\mathrm{D}}^{30}=-12.56(\mathrm{c}, 0.1, \mathrm{DMSO}), R_{\mathrm{f}}=0.12$. Found: $\mathrm{C} 64.40, \mathrm{H} 4.99, \mathrm{~N} 8.35 \% . \mathrm{C}_{27} \mathrm{H}_{25} \mathrm{~N}_{3} \mathrm{O}_{7}(503.5)$ requires $\mathrm{C}$ 64.41, H 5.00, N $8.35 \%$. m/z (EI-MS): 504 (33\%, M), 340 (100 $\%$, base peak), 218 (29\%), 180 (18\%), 122 (8\%), 79 (10\%). FTIR $v_{\max } \mathrm{cm}^{-1}: 3412.8$ (intramolecular $-\mathrm{OH}$, broad, stretch.), 2952.2 (aromatic str.), $1622.2(\mathrm{C}=\mathrm{N}), 1243.1(\mathrm{C}-\mathrm{N}) .{ }^{1} \mathrm{H}$ NMR $\delta_{\mathrm{H}}$ ppm: 3.2 $\left(1 \mathrm{H}, 2^{\prime} \mathrm{H}\right), 3.3\left(\mathrm{dd}, 1 \mathrm{H}, 3^{\prime} \mathrm{H}\right), 3.2\left(1 \mathrm{H}, 4^{\prime} \mathrm{H}\right), 3.5\left(1 \mathrm{H}, 5^{\prime} \mathrm{H}\right) .6 .1$ (d, $1 \mathrm{H}, J_{1,2}=8.2 \mathrm{~Hz}, 1$ 'H, anomeric proton), 6.4-7.8 (H, Ar- $\left.\mathrm{H}\right) .{ }^{13} \mathrm{C}$ NMR $\delta_{c}$ ppm: 156, 150.1, 144.0, 140.2,139.1, 138.5, 137.0, 135.0, 133.6, 130.2, 129.0, 128.6, 127.4, 127.0, 126.2, 125.8, 125.1, 124.9, $124.5,101.4,92.1,87.0,85.2,76.3,75.4,65.4$.

2-(3,4,5-Trimethoxyphenyl)-4,5-diphenyl-1-(N- $\beta$-D-glucopyranosyl)imidazole, 3e. Yield $62 \%,[\alpha]{ }^{30}=-16.30$ (c, 0.1, DMSO), $R_{\mathrm{f}}=0.14$. Found: C 65.70, H 5.89, N $5.12 \% . \mathrm{C}_{30} \mathrm{H}_{32} \mathrm{~N}_{2} \mathrm{O}$ (548.58) requires C 65.68, H 5.88, N $5.11 \% . m / z$ (EI-MS): 550 (12\%, M), 385 (100\%, base peak), 218 (19\%), 178 (21\%), 167 (11\%), 77 (18\%). FT-IR $v_{\max } \mathrm{cm}^{-1}: 3410.2$ (intramolecular -OH, broad, stretch.), 2954.0 (aromatic str.), $1625.4(\mathrm{C}=\mathrm{N}), 1244.4(\mathrm{C}-$ N). ${ }^{1} \mathrm{H}$ NMR $\delta_{\mathrm{H}}$ ppm: $3.2\left(1 \mathrm{H}, 2{ }^{\prime} \mathrm{H}\right), 3.5\left(\mathrm{dd}, 1 \mathrm{H}, 3{ }^{\prime} \mathrm{H}\right), 3.6(1 \mathrm{H}$, 4'H), 3.5(1H, 5'H). 6.4 (d, $1 \mathrm{H}, J_{1,2}=8.0 \mathrm{~Hz}, 1^{\prime} \mathrm{H}$, anomeric proton), 6.6-7.9 (H, Ar-H). ${ }^{13} \mathrm{C}$ NMR $\delta_{c}$ ppm: $153,151.1,151.0,139.3,138.4$ 137.2, 136.0, 135.7, 134.3, 131.8, 130.8, 130.2, 129.2, 128.4, 127.4, $127.1,126.3,125.6,125.0,124.2,124.0,104.1,85.3,76.0,75.2,65.0$, $57.8,57.0,56.0$.

2-(4-Fluorophenyl)-4,5-diphenyl-1-(N- $\beta$-D-glucopyranosyl)imidazole, $3 f$. Yield $78 \%,[\alpha]^{30}=-18.13$ (c, 0.1, DMSO), $R_{\mathrm{f}}=0.12$. Found: $\mathrm{C} 68.06, \mathrm{H} 5.29, \mathrm{~N} 5.89 \% . \mathrm{C}_{27} \mathrm{H}_{25} \mathrm{FN}_{2} \mathrm{O}_{5}(576.50)$ requires C 68.06, H 5.29, F 3.99, N $5.88 \%$. m/z (EI-MS): 476 (100 $\%$, M, base peak), 314 (35\%), 180 (26\%), 137 (12\%), 78 (15\%). FT-IR $v_{\max } \mathrm{cm}^{-1}: 3434.0$ (intramolecular $-\mathrm{OH}$, broad, stretch.), 
<smiles>[R]CNC(C)=O</smiles><smiles>[R]c1nc(-c2ccccc2)c(-c2ccccc2)[nH]1</smiles>

Scheme 1.

2948.8 (aromatic str.), $1635.3(\mathrm{C}=\mathrm{N}), 1239.8(\mathrm{C}-\mathrm{N}) .{ }^{1} \mathrm{H}$ NMR $\delta_{\mathrm{H}}$ ppm: $3.2\left(1 \mathrm{H}, 2^{\prime} \mathrm{H}\right), 3.0\left(\mathrm{dd}, 1 \mathrm{H}, 3^{\prime} \mathrm{H}\right), 3.4\left(1 \mathrm{H}, 4^{\prime} \mathrm{H}\right), 3.5\left(1 \mathrm{H}, 5^{\prime} \mathrm{H}\right)$. $6.1\left(\mathrm{~d}, 1 \mathrm{H}, J_{1,2}=8.5 \mathrm{~Hz}, 1^{\prime} \mathrm{H}\right.$, anomeric proton), 6.2-7.7 $(\mathrm{H}, \mathrm{Ar}-$ H). ${ }^{13} \mathrm{C}$ NMR $\delta_{\mathrm{c}} \mathrm{ppm}: 163,152.1,141.0,140.2,138.0,137.3,135.3$, $135.8,134.2$, 130.7, 130.6, 130.0, 129.2, 128.2, 127.8, 127.0, 126.2, 125.7, 125.2, 124.8, 123.0, 103.0, 85.0, 76.0, 75.4, 65.3.

2-(Furan-2-yl)-4,5-diphenyl-1-(N- $\beta$-D-glucopyranosyl)imidazole, $3 g$. Yield $65 \%,[\alpha]_{\mathrm{D}}^{30}=-8.10(\mathrm{c}, 0.1, \mathrm{DMSO}), R_{\mathrm{f}}=0.12$. Found: C 66.96, H 5.39, N 6.25\%. $\mathrm{C}_{25} \mathrm{H}_{26} \mathrm{~N}_{2} \mathrm{O}_{6}(448.47)$ requires $\mathrm{C}$ 66.95, H 5.39, N $6.25 \%$. m/z (EI-MS): 448 (11\%, M), 285 (100 $\%$, base peak), 178 (45\%), 107 (18\%), 78 (10\%), 67 (16\%). FTIR $v_{\max } \mathrm{cm}^{-1}: 3411.3$ (intramolecular-OH, broad, stretch.), 2934.1 (aromatic str.), $1632.0(\mathrm{C}=\mathrm{N}), 1240.2(\mathrm{C}-\mathrm{N}) .{ }^{1} \mathrm{H}$ NMR $\delta_{\mathrm{H}}$ ppm: 3.2 (1H, 2'H), 3.1 (dd, 1H, 3'H), 3.6 (1H, 4'H), 3.5 (1H, 5'H), 6.2 (d, 1H, $J_{l, 2}=8.4 \mathrm{~Hz}, 1^{\prime} \mathrm{H}$, anomeric proton), 6.4-7.9 (H, Ar-H). ${ }^{13} \mathrm{C} \mathrm{NMR} \delta_{\mathrm{c}}$ ppm: $154,144.0,140.2,138.1,133.3,132.0,131.8,131.0,130.7,130.4$ $130.0,129.1,128.1,127.8,127.4,126.0,125.6,125.3,124.0,123.4$, $121.2,104.0,85.3,77.0,75.4,65.3$.

\section{Results and Discussion}

Our research envisage starts from the synthesis of aglycons, i.e. 2-aryl-4,5-diphenyl- $1 H$-imidazoles, which were prepared following Radiszewski method by condensation of benzil, substituted-aldehyde and ammonium acetate in the acetic acid medium ${ }^{[21]}$ (Scheme 1). The series of these aglycons was prepared by changing substituents at position 2 (1a-g). The glucosylation was carried out using the known method. ${ }^{[22]}$

$\mathrm{N}$-Glucosylation proceeds similarly to $O$-glucosylation. Acetobromoglucose (ACBG) was used as a glucosyl donor for $\mathrm{N}$-glucosylation. 2-Aryl-4,5-diphenyl- $1 H$-imidazoles (1a-g) were allowed to condensed with acetobromoglucose in dioxane medium uynder reflux to afford 2-aryl-4,5diphenyl-1-( $N$ - $\beta$ - $D-2,3,4,6$-tetra- $O$-acetylglucopyranosyl)- imidazoles (2a-g) (Scheme 2). IR spectrum of $\mathbf{2 a}$ shows the following characteristic bands: 2962.3-3000, $1657(\mathrm{C}=\mathrm{C})$, 1576.2 (C=N, str.). ${ }^{1} \mathrm{H}$ NMR: 2.04, 1.94, 1.98, 2.01 (s, 3H, $\left.\mathrm{COCH}_{3}\right), 5.80(\mathrm{~d}, 1 \mathrm{H}$, anomeric proton), 6.2-7.8 (m, $15 \mathrm{H}$, Ar-H). These data indicate the presence of glucosyl group and absence of the secondary amine group, what confirms the formation of $N$-glucoside. The further deacetylation by sodium methoxide in methanol gives 2-aryl-4,5diphenyl-1-( $N-\beta-D$-glucopyranosyl)imidazoles (3a-g), the formation of which is confirmed by IR spectroscopy. Thus, a broad band at $3403.9 \mathrm{~cm}^{-1}$ (intramolecular $-\mathrm{OH}$, broad, stretch.) indicates the presence of carbohydrate's hydroxyl groups; as well as $1611.7(\mathrm{C}=\mathrm{N}), 1248.0(\mathrm{C}-\mathrm{N})$ bands and a band of $\beta$ - $D$-glucopyranosyl ring observed at $1029.3 \mathrm{~cm}^{-1}$ are associated with $N$-glucoside. ${ }^{1} \mathrm{H}$ NMR spectrum reveals the sugar proton signals between 2.30 to $4.38 \mathrm{ppm}$. The $\beta$-anomeric configuration was established by the appearance of the doublet at $\delta 5.9 \mathrm{ppm}$. Aromatic ring protons were observed between 6.95 to $8.06 \mathrm{ppm}$. In ${ }^{13} \mathrm{C}$ NMR spectrum of $\mathbf{3 a}$ the sugar C-1 atom consistently with 1,2-trans diaxial configiration gives the resonance signal near $104.04 \mathrm{ppm}$, i.e. downfield from signals of another glucosyl carbons appearing from 55.27 to 79.14 ppm. The resonance signals of the aromatic carbons (C-1 to C-18) and imidazole carbons are observed at 125.0 - 135.0 ppm and 135.0 - 155.0 ppm regions, correspondingly. In the mass spectra the protonated molecular ion peak as well as other important fragmentation peaks with their relative abundance are appeared at 458 (M, 10\%), 295 (base peak, $100 \%), 118$ (3\%), 78 (2\%).The compounds are semi-solid in nature. Due to the neighboring group participation only the $\beta$-form is obtained.<smiles>[R]c1nc(-c2ccccc2)c(-c2ccccc2)[nH]1</smiles>

$1 \mathrm{a}$

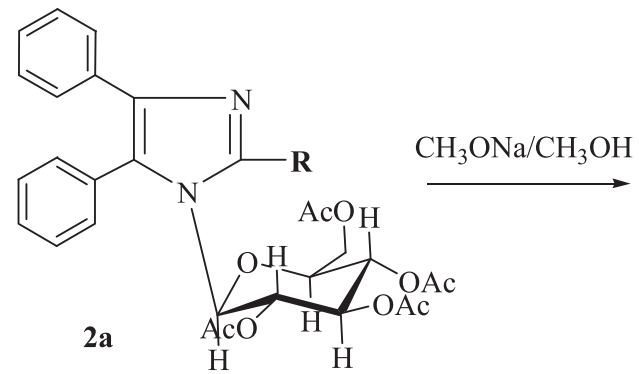

$\mathbf{R}$

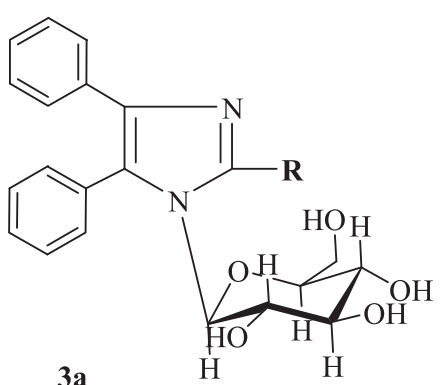

3a
d. 3-Nitrophenyl e. 3,4,5-Trimethoxyphenyl
f. 4-Flourophenyl g. Furyl
a. Phenyl
b. 4-Chlorophenyl
c. 2-Methoxyphenyl

Scheme 2. 
Acknowledgement. The authors are thankful to the Director, Sophisticated Analytical Instrument Facility (SAIF) Chandigarh, IIT-Powai Mumbai for providing the necessary spectral analysis, Head Department of Chemistry for providing necessary laboratory facilities and Head Department of Pharmacy for the biological study.

\section{References}

1. Kondo H., Taguchi M., Inoue Y., Sakamoto F., Tssukamoto G., J. Med. Chem. 1990, 33, 2012.

2. Dickens J. P., Ellames G. J., Hare N.J., Lawson K.R., McKay W.R., Mutters A.P., Myers P.L., Pope A.M.S., Upton R.M. J. Med. Chem. 1991, 34, 2356.

3. Ogata M., Matsumoto H., Hamada Y., Takehara M., Tawara K. J. Med. Chem. 1983, 26, 768.

4. Bhatia M., Naithani P.K., Bhalla T.N., Saxena A.K. J. Indan Chem. Soc. 1992, 60, 594.

5. Rama Sarma G.V.S, Reddy V.M. J. Indian Heterocycl. Chem. 1993, 3, 111.

6. Carini D.J., Dunica J.V., Aldrich P.E,. Chiu A.T., Johnson A.L., Pierce M.E., Price W.A., Santella III J.B., Wells G.J., Wexler R.R., Wong P.C., Yoo S.E., Pieter B.M., Timmermans W.M. J. Med. Chem. 1991, 34, 2525.

7. Bansal R.K Heterocyclic Chemistry (third edition) New Age International Publications, 1999.

8. Ganellin C.R. J. Med. Chem. 1973, 16, 620.

9. Eiseberg M.A. Biochem. J. 1966, 101, 598.
10. Zha C., Brown G.B., Brouillette W.J. J. Med. Chem. 2004, 47, 6519 .

11. Ben-Bassat A.A., Lavie D., Edery H., Porath G. J. Med. Chem. 1976, 19, 928.

12. Foye W.O., Tovivich P. J. Pharm. Sciences 1977, 66, 1607.

13. El-Sayed H.A., Moustafa A.H., Haikal A.Z., Abdou I.M., ElAshry E.S.H. Nucleosides, Nucleotides and Nucleic Acids 2008, 27, 1061.

14. Libnow S., Hein M., Harms M., Wende K., Lalk M., Reinke H., Langer P. J Carbohyd. Chem. 2009, 28, 483.

15. Ingle V.N., Hatzade K.M., Taile V.S., Gaidhane P.K., Kharche S.T. J. Carbohyd. Chem. 2007, 26, 107.

16. Hatzade K.M., Taile V.S., Gaidhane P.K., Haldar A.G.M., Ingle V.N. Ind. J. Chem. 2008, 47B, 1260.

17. Hatzade K.M., Taile V.S., Gaidhane P.K., Haldar A.G.M., Ingle V.N. Ind. J. Chem. 2009, 48B, 1548.

18. Taile V., Hatzade K., Gaidhane P., Ingle V. Turk. J. Chem. 2009, 33, 295.

19. Taile V.S., Hatzade K.M., Gaidhane P.K., Ingle V.N. J. Heterocycl. Chem. (2010) (in press).

20. Ingle V.N., Gaidhane P.K., Hatzade K.M., Umare V.D., Taile V.S. Int. J. Pharm. Tech. Res. 2009, 1, 605.

21. Steck E.A., Day A.R. J. Amer. Chem. Soc. 1943, 65, 452.

22. Howard G.A., Lythgoe B., Todd A.R. J. Chem. Soc. 1947, 1052.

23. Siddqui S.A., Narkhede U.C., Palimkar S.S., Daniel T., Lahoti R.J., Srinivasan K.V. Tetrahedron 2005, 61, 3539.

24. Sangshetti J.N., Kokare N.D., Kothakar S.A., Shinde D.B. Mont. Fur. Chem. 2008, 139, 125.

Received 08.05.2010

Accepted 23.06.2010

First published on the web 02.07.2010 